\title{
Model-Based Characterization of Thin Layers Using Pulse-Echo Ultrasound
}

\author{
Fredrik Hägglund ${ }^{1}$, Jesper Martinsson ${ }^{1}$, Johan E. Carlson ${ }^{1}$, and Carl Carlander ${ }^{2}$ \\ ${ }^{1}$ EISLAB, Dept. of Computer Science and Electrical Engineering, \\ Luleå University of Technology, SE-971 87 Luleå, Sweden, Fredrik.Hagglund@1tu.se \\ ${ }^{2}$ D-Flow Technology AB, Aurorum 2, SE-977 75 Luleå, Sweden.
}

\begin{abstract}
Measurements performed on a thin multilayered structure will imply a received signal waveform consisting of reverberant overlapping echoes. In this paper the multi-layered structure is modeled by a physical model and the Maximum Likelihood Estimator (MLE) is derived for the model parameters. A general recursive expression for the model is given. The model is evaluated using measurements on a thin three-layered structure, where two glass layers are bonded together. We show that measured signal waveforms can be reconstructed using the estimated parameters, and that physical properties can be extracted from the estimated model parameters. Simulations also show that physical parameters can be estimated for thicknesses of the bonding layer down to $50 \mu \mathrm{m}$ for a wavelength of $200 \mu \mathrm{m}$ of the ultrasonic pulse.
\end{abstract}

Keywords: Material characterization, multi-layered structure, parametric model, pulse-echo ultrasound

\section{A. Introduction}

Non-destructive testing of materials using ultrasound is considered a valuable tool to characterize thin layers in a multi-layered material. In this paper we propose a method to estimate material properties of the thin bonding layer in a multi-layered material using a physical model. The thinlayered material structure gives rise to overlapping ultrasonic echoes. The model structure is therefore chosen so that the variations in the received ultrasonic signal waveform are captured by a small number of model parameters.

The material is modeled using a continuous autoregressive (AR) model with parameters connected to physical properties, related to the thickness of the layers, the reflection and the transmission coefficients given by the boundaries between the layers, and the attenuation inside the layers. The paper derives the Maximum Likelihood Estimator (MLE) for the model parameters.

We show with simulations how accurate estimates of the model parameters we get when the thicknesses of the layers decrease. Material properties such as speed of sound, acoustic impedance, and density of the layers can be estimated from the parameters with corresponding confidence interval for varying signal-to-noise ratio and material layer properties.

The model is evaluated with real measurements on two glass layers bonded together by a thin layer, where some properties of the layers are known. The measured signal waveform consists of reverberant overlapping echoes, and using the proposed parametric model we show that reliable estimates of the bonding layer properties can be deduced from the estimated parameters.

\section{B. Theory}

In this section, the signal model and the parameter estimation for the property extraction problem is stated in the frequency domain. The physical properties related to the estimated parameters are stated and the parameter estimation is explained.

\section{B.1. General Parametric Model}

Sending an ultrasonic echo through a multi-layered material will produce a received signal waveform consisting of several delayed and attenuated echoes. A structure consisting of thin layers will produce reverberating echoes due to multiple reflections, resulting in an overlapping signal waveform at the receiver. The modeling of a multilayered structure is performed by a physical model of the layers, using a continuous AR model. The parameters in the model are the reflection coefficients, the time of flight of the pulse in the layers, and a parameter directly related to the attenuation coefficient for each layer. These parameters give us the structure of the multi-layered material. From this physical model of the multi-layered structure it is then possible to extract material properties.

An iterative model for the reflection from a multilayered structure has been derived in [1], but is in [2] derived in a more convenient way for system transfer function identification. The model of the transfer function is a reverberant multi-layered structure $\mathrm{H}_{t f}(\omega)$. The output $\mathrm{Y}(\omega)$, by using $\mathrm{U}(\omega)$ as the input signal and assuming linear acoustics, is then represented as

$$
\mathrm{Y}(\omega)=\mathrm{H}_{t f}(\omega) \mathrm{U}(\omega)
$$

where

$$
\mathrm{H}_{t f}(\omega)=\left(R_{01}+\mathrm{H}(\omega)\right) e^{-j \omega \tau_{0}},
$$

where $j=\sqrt{-1}, \omega$ is the angular frequency, $R_{01}$ is the reflection coefficient from the top layer, and $\tau_{0}$ is the time delay for the ultrasonic pulse traveling the distance $d_{0}$ back and forward from the transducer to the material in the water buffer region. The expression $H(\omega)$ in (2) is the general parametric model for a multi-layered structure, and 
$\mathrm{H}(\omega)=\mathrm{H}_{q_{0}}(\omega)$, where $q_{0}$ is the total number of layers. The recursive expression starting at $q=q_{0}$ is then expressed as

$$
\left\{\begin{array}{lll}
\mathrm{H}_{q}(\omega)=A_{q+1}+\frac{B_{q+1} C_{q+1} \mathrm{H}_{q-1}(\omega)}{1-D_{q+1} \mathrm{H}_{q-1}(\omega)} & , q>1 \\
\mathrm{H}_{q}(\omega)=A_{q+1} & , q=1
\end{array}\right.
$$

where the expressions for $A, B, C$ and $D$ are;

$$
\begin{aligned}
A_{q} & =\frac{T_{Q-1, Q} R_{Q, Q+1} T_{Q, Q-1} e^{-j \omega \tau_{Q}} G_{Q}(\omega)}{1-R_{Q, Q-1} R_{Q, Q+1} e^{-j \omega \tau_{Q}} G_{Q}(\omega)}, \\
B_{q} & =\frac{T_{Q, Q-1}}{1-R_{Q, Q-1} R_{Q, Q+1} e^{-j \omega \tau_{Q}} G_{Q}(\omega)}, \\
C_{q} & =\frac{T_{Q-1, Q} e^{-j \omega \tau_{Q}} G_{Q}(\omega)}{1-R_{Q, Q-1} R_{Q, Q+1} e^{-j \omega \tau_{Q}} G_{Q}(\omega)}, \\
D_{q} & =\frac{R_{Q, Q-1} e^{-j \omega \tau_{Q}} G_{Q}(\omega)}{1-R_{Q, Q-1} R_{Q, Q+1} e^{-j \omega \tau_{Q}} G_{Q}(\omega)},
\end{aligned}
$$

where the subscript $Q$ is connected to $q$ as

$$
Q=q_{0}-q+2
$$

The expression $G(\omega)$ is the frequency dependent attenuation in the material in the specific layer. The attenuation can be described as the pressure change of a wave due to absorption and scattering and is expressed as,

$$
P_{x}=P_{0} e^{-\alpha_{s}(\omega) x},
$$

where $P_{0}$ is the pressure at some location and $P_{x}$ is the reduced pressure at distance $x$ from the initial location. The quantity $\alpha_{s}(\omega)$ is the frequency dependent attenuation coefficient expressed in nepers $/ \mathrm{m}$. Given that $x=2 d$, where $d$ is the thickness of the specific layer, and that the attenuation coefficient is proportional to the square of the sound frequency, $\alpha_{s}(\omega)=\tilde{\alpha} \omega^{2}$, the frequency dependent attenuation in the model is defined as

$$
G(\omega)=e^{-\tilde{\alpha} \omega^{2} 2 d},
$$

where $\tilde{\alpha}$ is a constant containing several attenuation effects, e.g. the classical absorption [3]. Extending the parametric model with the attenuation inside the layers provide possibilities to capture more of the signal waveform. Using (3) the model for an arbitrary number of layers can be derived.

\section{B.2. Model for Three Layers}

The experimental setup shown in Fig. 1 motivates the selection of a three layer model. In this specific experiment, two glass layers were bonded together by a thin bonding layer, meaning that the first and the third layer should have the same properties. This implies that the general model can be simplified before applied on this specific problem. In this case we have;

$$
\begin{aligned}
R_{23} & =-R_{12}, \\
R_{34} & =-R_{01}, \\
\tilde{\alpha}_{3} & =\tilde{\alpha}_{1},
\end{aligned}
$$

and using the relationship between the reflection and transmission coefficients

$$
\begin{aligned}
R_{l, k} & =-R_{k, l}, \\
T_{k, l} & =1+R_{k, l},
\end{aligned}
$$

we can define an analytical expression for the transfer function in (2).

Our only received information about the layered material, in addition to some pre-knowledge of the material properties, is the signal waveform, see Fig. 1. However, to perform the parameter estimation and the system identification we require an input signal. If the first echo in our received signal waveform is separable from the rest of the waveform, we can use a scaled version of this echo as the input signal. The first echo is then used to create the input signal as

$$
\mathrm{U}(\omega)=\frac{1}{R_{01}} e^{j \omega \tau_{0}} \tilde{\mathrm{Y}}(\omega) .
$$

On the other hand, if the first echo is not separable from the waveform, the input signal must be captured from a separate measurement on a thicker layer of the same medium, see Fig. 4a, to get $\tilde{Y}(\omega)$. The input signal is captured by windowing and by using (16) after an appropriately scaling and a pre-alignment before the estimation is performed.

The parameterized transfer function $\mathrm{H}_{t f}(\omega, \theta)$ in (2) (omitting $\omega$ for notational simplicity) is then defined as

$$
\mathrm{H}_{t f}(\theta)=\left(R_{01}+\frac{E}{F}\left(1-R_{01}^{2}\right) e^{-j \omega \tau_{1}} G_{1}\right) e^{-j \omega \tau_{0}},
$$

where

$$
\begin{aligned}
E= & \left(R_{12}-R_{12} e^{-j \omega \tau_{2}} G_{2}+R_{01} R_{12}^{2} e^{-j \omega \tau_{3}} G_{3}\right. \\
& \left.-R_{01} e^{-j \omega\left(\tau_{2}+\tau_{3}\right)} G_{2} G_{3}\right) \\
F=( & +R_{01} R_{12} e^{-j \omega \tau_{1}} G_{1}-R_{12}^{2} e^{-j \omega \tau_{2}} G_{2} \\
& +R_{01} R_{12} e^{-j \omega \tau_{3}} G_{3}+R_{01}^{2} R_{12}^{2} e^{-j \omega\left(\tau_{1}+\tau_{3}\right)} G_{1} G_{3} \\
& -R_{01} R_{12} e^{-j \omega\left(\tau_{2}+\tau_{3}\right)} G_{2} G_{3} \\
& -R_{01} R_{12} e^{-j \omega\left(\tau_{1}+\tau_{2}\right)} G_{1} G_{2} \\
& \left.-R_{01}^{2} e^{-j \omega\left(\tau_{1}+\tau_{2}+\tau_{3}\right)} G_{1} G_{2} G_{3}\right)
\end{aligned}
$$

and where $\theta=\left[\begin{array}{lllllll}R_{01} & R_{12} & \tau_{1} & \tau_{2} & \tau_{3} & \tilde{\alpha}_{1} & \tilde{\alpha}_{2}\end{array}\right]^{T}$ is the parameter vector.

\section{B.3. Parameter Estimation}

Deriving the MLE for the parameters in the signal model described in the previous sections is performed in the frequency domain. The noise on both the input signals and the output signals is assumed to be white and Gaussian, implying that the MLE ends up in a Nonlinear Least Squares (NLS) problem. To achieve a faster iteration in the minimization process, analytical expressions for the gradient and the Hessian is derived. The Gauss-Newton (GN) linearization method is used [4]

$$
\hat{\theta}_{k+1}=\hat{\theta}_{k}-\left(\Re\left\{\frac{\partial \epsilon^{H}}{\partial \theta_{k}} \frac{\partial \epsilon}{\partial \theta_{k}}\right\}\right)^{-1} \cdot \Re\left\{\frac{\partial \epsilon^{H}}{\partial \theta_{k}} \epsilon\right\},
$$

where $\epsilon(\omega, \theta)$ is the scaled error function given in [5]. In addition to the GN algorithm, the Hessian in (20) is also regularized. 


\section{B.4. Physical Properties}

The estimated parameter vector $\hat{\theta}$, given by the model, can be used to directly extract several properties, such as speed of sound, acoustic impedance, and the density. Assuming that the thicknesses of the layers are known, the speed of sound can be estimated as

$$
\hat{c}=\frac{2 d}{\hat{\tau}},
$$

where $\hat{\tau}$ is the estimated time of flight taken from the parameter vector $\hat{\theta}$. The frequency dependent part of the speed of sound is neglected due to the very short relaxation time. The acoustic impedance can be estimated as,

$$
\hat{z}_{l}=\hat{z}_{l-1}\left(\frac{1+\hat{R}_{l-1, l}}{1-\hat{R}_{l-1, l}}\right),
$$

where $\hat{R}_{l-1, l}$ is the estimated reflection coefficient from the boundaries between the layers, $\hat{z}_{l-1}$ is the estimated impedance in the previous layer and finally for $l=0$ the acoustic impedance in the water buffer region is defined as

$$
z_{0}=\rho_{0} c_{0},
$$

where both the density in water, $\rho_{0}$, and the speed of sound in water, $c_{0}$, is assumed to the known, see [3]. The density can be estimated as,

$$
\hat{\rho}=\frac{\hat{z}}{\hat{c}}
$$

by using (21) and (22).

\section{Experiment Setup}

All measurements were conducted with the ultrasound transducer immersed in water and with the specimen surrounded by water. The measurements were performed in a pulse-echo configuration, see Fig. 1. The temperature in the water was kept constant at $19.85{ }^{\circ} \mathrm{C}$ to within $\pm 0.05^{\circ} \mathrm{C}$.

The examined specimen consisted of two pyrex glass layers bonded together by a thin bonding layer. The thickness of the top glass layer was $1.83 \mathrm{~mm}$ and the thickness of the bottom glass layer was $3.78 \mathrm{~mm}$. The thickness of the bonding layer was $0.53 \mathrm{~mm}$ and two different materials were used as bonding material, construction silicone and epoxy.

For all the experiments performed in this work, a $5 \mathrm{MHz}$ unfocused piezoelectric transducer (V3456) from Panametrics with an element size of $13 \mathrm{~mm}$ was used. To excite and receive acoustic pulses, a Panametrics Pulser/Receiver Model 5073PR, operating in pulse-echo mode was used. The acquired ultrasonic echoes were sampled using a Gage CompuScope CS12400, at $200 \mathrm{MHz}$ with a 12-bit resolution.

\section{Results}

\section{D.1. Simulation Results}

In the simulations we can investigate how accurate estimates of the parameters we can get for decreasing thickness of the bonding layer. All estimated parameters can be

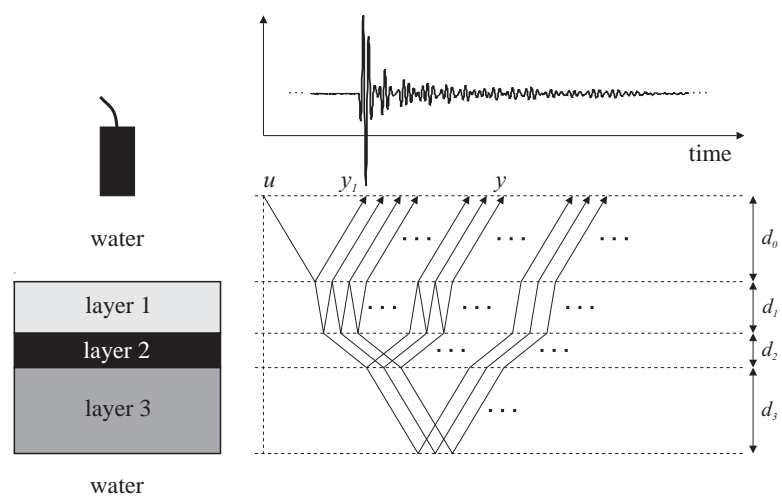

Fig. 1. The experimental setup in a pulse-echo configuration. The waveforms and the notations are in the time domain, where $u(t)$ represents the input signal, $y(t)$ is the entire output signal and $y_{1}(t)$ is the first echo in the output signal.

examined, but in this paper we present the results for one parameter. In Fig. $2 \mathrm{a}$ the estimated time of flight through the bonding layer, $\hat{\tau}_{2}$, is plotted together with the true, $\tau$, value for decreasing thickness $d_{2}$. A simulated output signal is used, where the initial values of the parameters are up to $5 \%$ from the values used to create the output signal. In Fig. $2 b$ the difference between the estimated and the true time of flight is plotted, and we get very certain estimates for thicknesses down to about $50 \mu \mathrm{m}$. Below that thickness the estimate is still quite accurate but somewhat more unstable, also seen in Fig. 2c, where the estimated standard deviation of the estimated time of flight is plotted for decreasing thickness. The standard deviation increases for thickness below $50 \mu \mathrm{m}$.
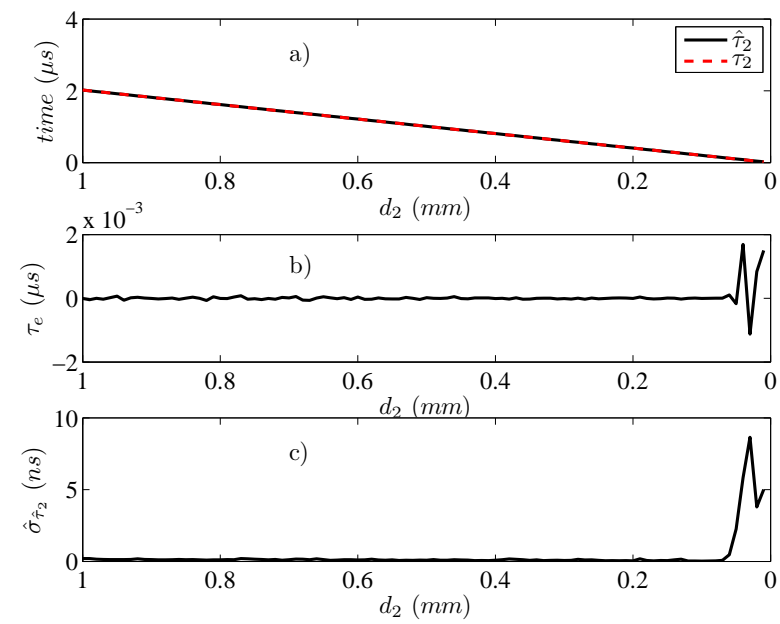

Fig. 2. In a) the estimated time of flight $\hat{\tau_{2}}$ (solid) and the true value $\tau_{2}$ (dotted) are plotted. In b) the difference $\tau_{e}=\hat{\tau}_{2}-\tau_{2}$ is plotted. In c) the estimated standard deviation of the estimated time of flight $\hat{\sigma}_{\hat{\tau}_{2}}$ is plotted. The $x$-axis is the thickness, $d_{2}$.

\section{D.2. Measurement Results}

The physical model is able to recreate the measured signal waveform from the seven parameters. When the received ultrasonic signal waveform have a separable first echo, that echo is also used as the echo creating the input 
signal, see Fig. 3. In this case the residual is approaching white noise. On the other hand, when the received ul-

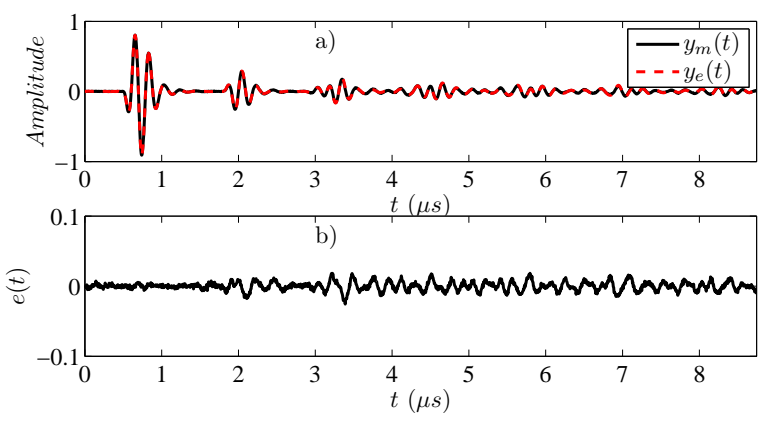

Fig. 3. In a) both the measured $y_{m}(t)$ and the estimated $y_{e}(t)$ signal waveforms are plotted, and in $\mathrm{b}$ ) the residual $e(t)=$ $y_{m}(t)-y_{e}(t)$ is plotted.

trasonic signal waveform contains nothing but overlapping echoes a separate measurement is required. In Fig. 4 the results from a measurement where epoxy was used as the bonding material is shown. In Fig. 4a, we have the separate measurement from where the input signal is created, and in Fig. 4b the measured and the estimated signal waveform is shown. The output signal and the estimated signal is apparently coinciding. When a separate measurement is performed to capture the input signal, we usually have a slight variation in the first echo, see Fig. 4c. This variation is usually due to distance and angle variations in the different measurements, and can be covered by statistical tools. These effects are neglected in this paper, thus we are strictly considering the hard physical model.
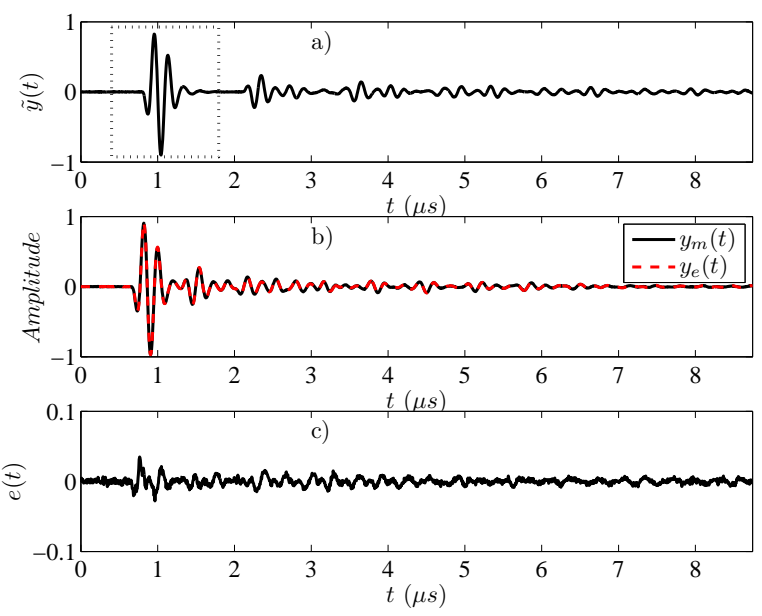

Fig. 4. In a) the separate measurement to extract the input echo is plotted. In b) the measured $y_{m}(t)$ and the estimated $y_{e}(t)$ output signals are plotted, and in c) the residual $e(t)=y_{m}(t)-$ $y_{e}(t)$ is plotted. Epoxy was used as bonding material.

In the parameter estimation algorithm an accurate initial guess is essential. Since this model is based on physical properties, we find the initial guess of the parameters from the theoretical values calculated from our pre-knowledge about the material. The mean of the estimated parameters is presented together with the estimated $2 \sigma$ interval for the parameters in Tab. 1. The estimated values of the parameters are very repeatable indicated by the low $2 \sigma$ intervals.

Table 1. The estimated parameters for two experiments.

\begin{tabular}{|c|c|c|}
\hline & Silicone & Epoxy \\
& mean $\pm 2 \sigma$ & mean $\pm 2 \sigma$ \\
\hline$\hat{R}_{01}$ & $0.811 \pm 0.4 \cdot 10^{-3}$ & $0.816 \pm 0.6 \cdot 10^{-3}$ \\
$\hat{R}_{12}$ & $-0.862 \pm 0.3 \cdot 10^{-3}$ & $-0.710 \pm 0.9 \cdot 10^{-3}$ \\
$\hat{\tau}_{1}$ & $0.63 \cdot 10^{-6} \pm 40 \cdot 10^{-12}$ & $0.63 \pm 65 \cdot 10^{-12}$ \\
$\hat{\tau}_{2}$ & $1.08 \cdot 10^{-6} \pm 86 \cdot 10^{-12}$ & $0.46 \pm 132 \cdot 10^{-12}$ \\
$\hat{\tau}_{3}$ & $1.30 \cdot 10^{-6} \pm 129 \cdot 10^{-12}$ & $1.30 \pm 169 \cdot 10^{-12}$ \\
$\hat{\tilde{\alpha}}_{1}$ & $177 \cdot 10^{-15} \pm 6.7 \cdot 10^{-15}$ & $89 \cdot 10^{-15} \pm 13 \cdot 10^{-15}$ \\
$\hat{\tilde{\alpha}}_{2}$ & $4778 \cdot 10^{-15} \pm 66 \cdot 10^{-15}$ & $15460 \cdot 10^{-15} \pm 164 \cdot 10^{-15}$ \\
\hline
\end{tabular}

From the estimated parameters we can extract some physical properties. In Tab. 2, the estimated speed of sound and the estimated density are presented for the two different bonding materials and also for the first glass layer. The exact mixture of silicone and epoxy are unknown and hence it is difficult to validate the results. However, the properties for the glass layer agree with previous work, see e.g. [6].

Table 2. Properties extracted from the parameters.

\begin{tabular}{|c|c|c|c|}
\hline & Silicone & Epoxy & Glass pyrex \\
& mean $\pm 2 \sigma$ & mean $\pm 2 \sigma$ & mean $\pm 2 \sigma$ \\
\hline$\hat{c}(\mathrm{~m} / \mathrm{s})$ & $984 \pm 0.1$ & $2314 \pm 0.7$ & $5798 \pm 0.5$ \\
$\hat{\rho}\left(\mathrm{kg} / \mathrm{m}^{3}\right)$ & $1073 \pm 3.2$ & $1066 \pm 4.4$ & $2478 \pm 7.0$ \\
\hline
\end{tabular}

\section{E. Conclusions}

It has been shown with measurements that the parametric model presented in this paper is able to reconstruct the ultrasonic signal waveform consisting of multiple overlapping echoes from within multi-layered structures by using the estimated parameter vector. Given the model and its parameters, it is possible to extract material properties of the individual layers.

\section{F. Literature}

[1] L. M. Brekhovskikh, Waves in Layered Media, vol. 2. Academic Press, 1980

[2] F. Hägglund, J. Martinsson, and J. E. Carlson, "Flaw Detection in Layered Media Based on Parametric Modeling of Overlapping Ultrasonic Echoes," in Proc. IEEE Int. Ultrason. Symp., IEEE, 2006.

[3] L. E. Kinsler, A. R. Frey, A. B. Coppens, and J. V. Sanders, Fundamentals of Acoustics. New York: Wiley, 3rd ed., 1982.

[4] S. M. Kay, Fundamentals of Statistical Signal Processing: Estimation Theory, vol. 1. Prentice Hall, 1993.

[5] R. Pintelon and J. Schoukens, System Identification: A Frequency Domain Approach, vol. 1. IEEE Press, 2001.

[6] C. Graciet and B. Hosten, "Simultaneous Measurement of Speed, Attenuation, Thickness and Density with Reflected Ultrasonic Waves in Plates," in Proc. IEEE Int. Ultrason. Symp., pp. 1219-1222, IEEE, 1994. 